A whole-head magnetoencephalography system with compact axial gradiometer structure

This article has been downloaded from IOPscience. Please scroll down to see the full text article.

2009 Supercond. Sci. Technol. 22045023

(http://iopscience.iop.org/0953-2048/22/4/045023)

The Table of Contents and more related content is available

Download details:

IP Address: 140.112.113.225

The article was downloaded on 24/07/2009 at 07:10

Please note that terms and conditions apply. 


\title{
A whole-head magnetoencephalography system with compact axial gradiometer structure
}

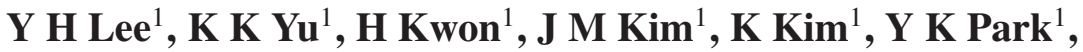

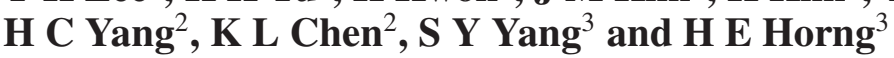 \\ ${ }^{1}$ Medical Metrology Center, Korea Research Institute of Standards and Science, Doryong 1, \\ Yuseong, Daejeon 305-340, Korea \\ ${ }^{2}$ Department of Physics, National Taiwan University, Taipei, Taiwan \\ ${ }^{3}$ Institute of Electro-Optical Science and Technology, National Taiwan Normal University, \\ Taipei, Taiwan \\ E-mail: yhlee@kriss.re.kr
}

Received 1 December 2008, in final form 20 January 2009

Published 17 March 2009

Online at stacks.iop.org/SUST/22/045023

\begin{abstract}
We have fabricated a whole-head superconducting quantum interference device (SQUID) gradiometer system for measuring the magnetoencephalography (MEG) of the human brain. Major technical features of the system are the compact structure of the gradiometer and compact readout electronics. As the gradiometers, first-order gradiometers of $50 \mathrm{~mm}$ baseline were used to reduce environmental noises. To simplify the fabrication process of the gradiometers, and to increase the refill interval of liquid $\mathrm{He}$, the superconductive connection between the pickup coil wires and input coil pads was done by direct bonding of $\mathrm{Nb}$ wires. Therefore, bulky superconducting blocks or superconducting screws were not used for the superconductive connection, and superconducting shielding was not used around the SQUID module, resulting in no distortion of external field uniformity. The distance between the compensation coil of the gradiometer and SQUID input coil pad was reduced to $10 \mathrm{~mm}$, and the total length of the gradiometer is $70 \mathrm{~mm}$. A sensor helmet having 128 gradiometers was cooled inside a helmet-shape liquid He dewar. The average boil-off rate of the MEG system is $101 \mathrm{~d}^{-1}$ and the refill interval is 7 days when the 128-channel system is in operation every day. To simplify the readout electronics of the SQUID system, double relaxation oscillation SQUIDs (DROSs) having large flux-to-voltage transfer coefficient were used. The magnetically shielded room (MSR) has a wall thickness of $200 \mathrm{~mm}$, and consists of two layers of Permalloy and one layer of aluminum. When the 128-channel system was operated inside the MSR, the average magnetic field noise level of the 128 channels was about $3.5 \mathrm{fT}_{\mathrm{rms}} \mathrm{Hz}^{-1 / 2}$ at $100 \mathrm{~Hz}$. Spontaneous and evoked brain magnetic fields were measured using the developed system.
\end{abstract}

(Some figures in this article are in colour only in the electronic version)

\section{Introduction}

Measurement of magnetic fields from the human brain, i.e., magnetoencephalography (MEG) technology, can provide useful information for the functional study of the human brain non-invasively [1-4]. By using MEG technology, mapping of neural currents with high temporal and spatial accuracy can be done. So far, several types of MEG system have been developed and they are used in hospitals or brain research institutes. To distinguish the weak brain magnetic signals from the strong environmental noises, an effective combination of a magnetically shielded room (MSR) and SQUID pickups is needed. Considering the high price of Nialloy materials used for the magnetic shielding, it is desirable to use gradiometers rather than magnetometers to reduce the amount of Ni-alloy. Typical gradiometers are axial pickup coils with $\mathrm{Nb}$ or $\mathrm{Nb}$-alloy wire wound on nonmagnetic coil bobbins [5, 6]. 
For the superconductive connection between the pickup coil and the input coil, superconducting $\mathrm{Nb}$ blocks or strips with screw terminals are usually used. To eliminate the pickup area of the magnetic field due to the superconducting connection structure, the $\mathrm{Nb}$ blocks are sometimes shielded using a superconducting tube [7-10]. This superconducting block and tube introduces distortion of the magnetic fields, and they are installed at a sufficiently large distance from the compensation coil of the gradiometer to maintain the balancing of the gradiometer against the external fields. The increased length of the gradiometer requires a higher level of liquid He to keep both the SQUID and the pickup coil superconducting. In order to increase the refill interval of the liquid $\mathrm{He}$, it is desirable to position the SQUIDs as close as possible to the gradiometers. In this paper, we introduce a novel superconductive connection method realized by direct bonding of $\mathrm{Nb}$ wire between the pickup coil wires and input coil pads, without using an intermediate bulky connection structure. Thus, the fabrication process of the gradiometer became simpler and the total length of the gradiometer was shortened.

A multi-channel SQUID system requires simple and compact room-temperature readout electronics. For the simple structure of the flux-locked loop (FLL) circuits, the output voltages and flux-to-voltage transfers of the SQUIDs should be large enough so that the contribution of preamplifier input noise is negligible in direct readout mode [11, 12]. A double relaxation oscillation SQUID (DROS), based on the relaxation oscillation of a hysteretic SQUID and a reference junction, provides large flux-modulated voltage output and steep fluxvoltage transfer coefficient $[13,14]$. In this study, we used DROSs and measured the voltage outputs of the DROSs directly using room-temperature preamplifiers, and made the FLL circuits compact.

\section{DROS axial gradiometer}

\subsection{Double relaxation oscillation SQUID sensor}

As a novel approach to increase the flux-to-voltage transfer, we used a double relaxation oscillation SQUID (DROS). A DROS consists of a signal SQUID and a reference junction in series, and is shunted by a relaxation circuit of a resistor and an inductor. The design of the signal SQUID was based on the Ketchen-type DC SQUID having a washer-type SQUID loop with a tightly coupled input coil [7]. The SQUID loop consists of two $130 \mu \mathrm{m} \times 130 \mu \mathrm{m}$ square holes connected in parallel, and the inductance of the signal SQUID is $144 \mathrm{pH}$. The signal SQUID has two Josephson junctions, each with a size of $4 \mu \mathrm{m} \times 4 \mu \mathrm{m}$, and the reference junction has a size of $5 \mu \mathrm{m} \times 5 \mu \mathrm{m}$. In contrast to DC SQUIDs, all the junctions in the DROS are hysteretic. When a DC bias current is applied, relaxation oscillations occur. If we measure the voltage across the reference junction, the DROS functions as a comparator of the two critical currents, the signal SQUID's critical current and the reference junction's critical current. To position the critical current of the reference junction near the midpoint of the modulation range of the signal critical current, the area of the reference junction $\left(25 \mu \mathrm{m}^{2}\right)$ is $78 \%$ of the sum of junction area in the signal SQUID $\left(32 \mu \mathrm{m}^{2}\right)$. The relaxation circuit has a resistance of $1.5 \Omega$ and an inductance of $2 \mathrm{nH}$, resulting in the relaxation time constant of $1.3 \mathrm{~ns}$.

The input coil consists of two series-connected coils, of 20 turns each with a line-width of $5 \mu \mathrm{m}$, integrated on each SQUID hole, and the input coil inductance was calculated to be $196 \mathrm{nH}$. The mutual inductance between the input coil and the SQUID was calculated to be $4.78 \mathrm{nH}[15,16]$. A feedback coil was formed in the flux transformer circuit so that the external feedback scheme was used to eliminate magnetic coupling between neighboring pickup coils [17]. In order to remove any trapped fluxes in the SQUID or flux transformer, a $100 \Omega$ resistance heater was placed at a close distance from the SQUID.

The fabrication of DROS sensors was based on $\mathrm{Nb} / \mathrm{AlO}_{x} / \mathrm{Nb}$ junction technology using a simple four-level process, consisting of junction trilayer deposition by DC magnetron sputtering, junction definition by reactive ion etching, $\mathrm{SiO}_{2}$ insulation deposition by plasma-enhanced chemical vapor deposition, and $\mathrm{Nb}$ wiring deposition. After the $\mathrm{Nb}$ wiring deposition, a thin Pd film was deposited on the surface of the $\mathrm{Nb}$ to protect the $\mathrm{Nb}$ surface from oxidation and to facilitate a superconductive bonding. The size of each DROS sensor is $3 \mathrm{~mm} \times 6 \mathrm{~mm}$, and about 200 sensors were fabricated together in a 3 inch Si wafer, and three wafers were fabricated in a single run. The fabricated DROS sensors were mounted on printed circuit boards (PCBs) having a 8-pin connector.

\subsection{Axial first-order gradiometer}

The gradiometer is an axial pickup coil of first order, made by winding an insulated NbTi wire on a nonmagnetic coil bobbin made of fiberglass reinforced plastic (FRP). The diameter of the NbTi wire is $0.125 \mathrm{~mm}$, and the coil diameter is $20 \mathrm{~mm}$. The number of turns of the coils is 1 , both for the proximal and compensation coil. The baseline of the gradiometer is $50 \mathrm{~mm}$. For the superconductive connection between the pickup coil wires and input coil pads, the ends of the pickup coil wires were glued on the FRP bobbin surface and then the insulation layer was removed with a knife. During this process, the round ends of the NbTi wires became flat, so an ultrasonic bonder wedge can be pressed to this horizontally flat NbTi surface. Superconductive connection was made directly by using ultrasonic bonding of a $\mathrm{Nb}$ wire between the ends of the pickup coil wires and the input coil pads, as shown schematically in figure $1(\mathrm{a})$. Since $\mathrm{Nb}$ wire is a refractory material, it became ductile by applying a current through it in a vacuum chamber [18]. The Nb bonding wire used was $25 \mu \mathrm{m}$ in diameter.

Due to the compact structure of the superconductive connection, the field distortion and stray pickup area could be minimized. The stray pickup area was estimated to be about $0.3 \mathrm{~mm}^{2}$, which is about $0.1 \%$ of the area of the sending coil. This value is similar in magnitude to the unbalances of typical axial first-order gradiometers, and seems to be good enough for operation inside a moderately shielded room. Because of the negligible amount of field distortion due to the superconductive 


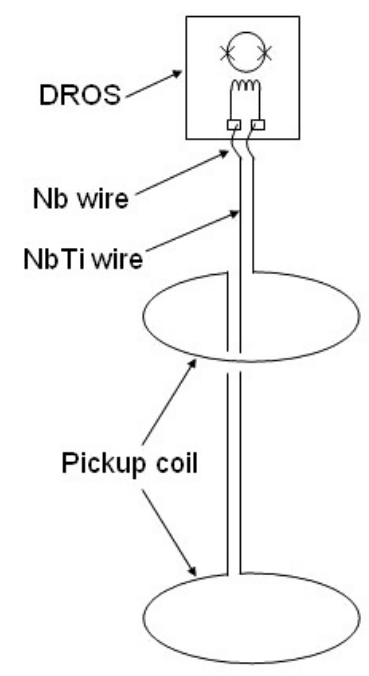

(a)

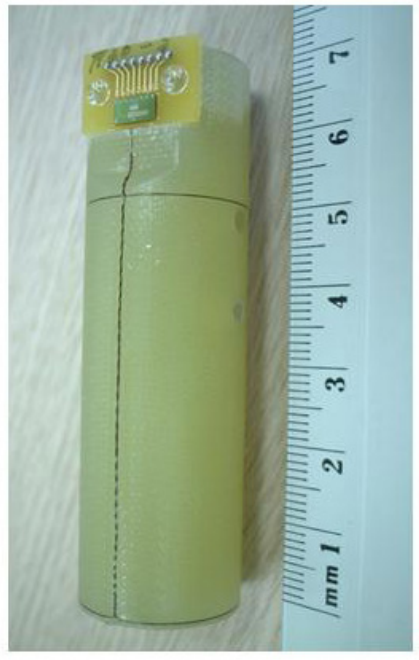

(b)
Figure 1. Structure of the axial gradiometer. (a) Schematic diagram of the gradiometer and (b) photograph of the fabricated gradiometer.

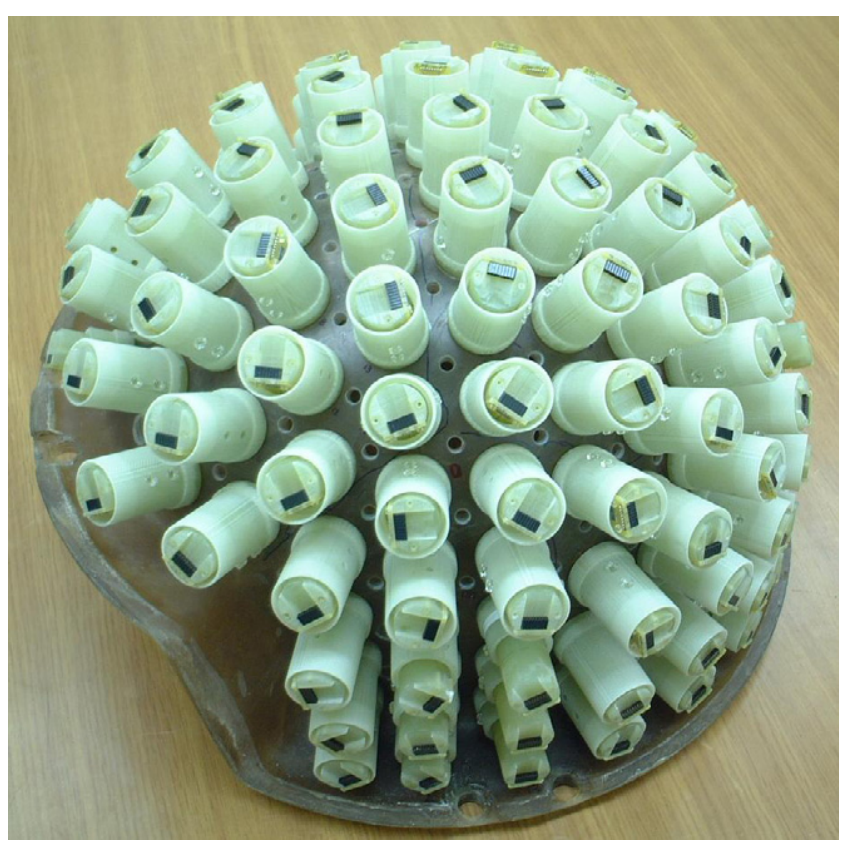

Figure 2. Layout of the helmet-type 128-channel axial gradiometer array.

connection structure, the SQUID sensor was placed close to the pickup coil, about $10 \mathrm{~mm}$ from the compensation coil, reducing the level of liquid He required to cool both the pickup coil and the SQUID sensor. The inductance of the pickup coil is $130 \mathrm{nH}$. The field-to-flux transfer coefficient of the gradiometer is $0.46 \mathrm{nT} / \Phi_{0}$. A fabricated gradiometer is shown in figure 1(b).

\section{Whole-head MEG system}

\subsection{Whole-head gradiometer insert}

The 128 axial gradiometers were distributed in a helmet configuration over the bottom of a helmet dewar. The sensor

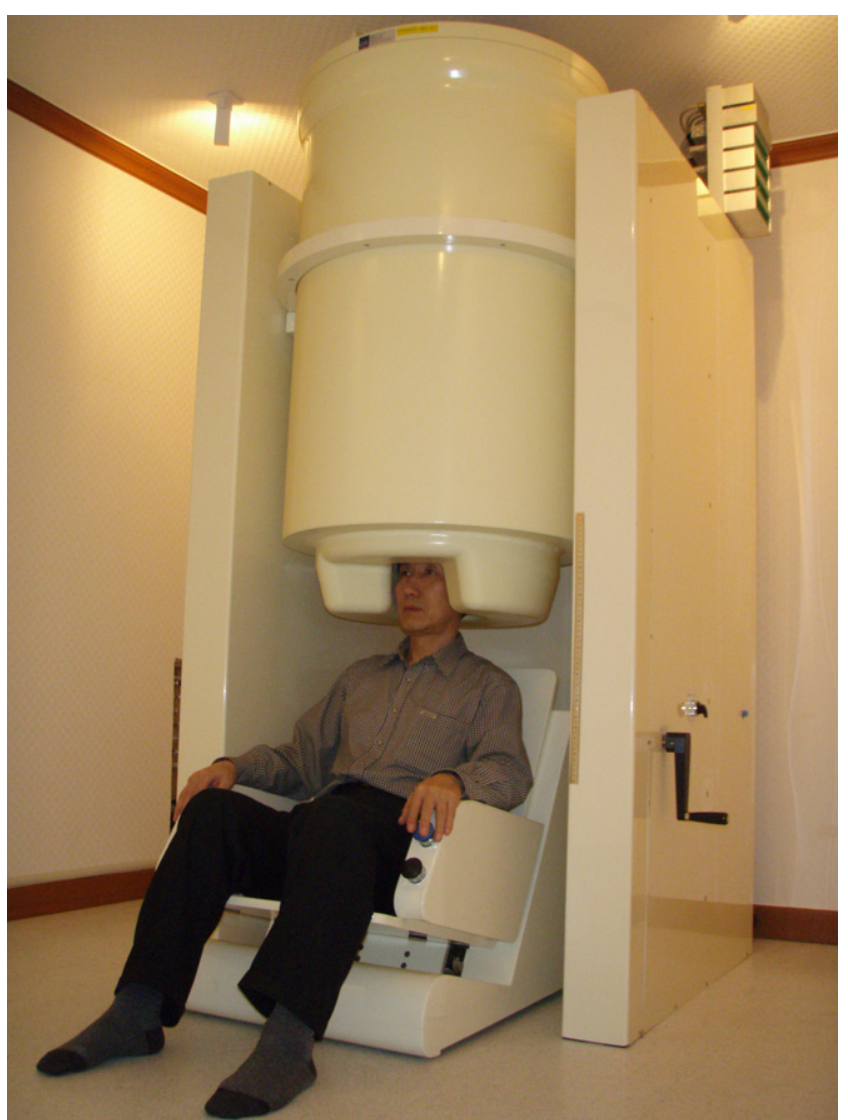

Figure 3. Photograph of the installed system. The nonmagnetic gantry is capable of vertical movement and tilting up to $30^{\circ}$. The subject chair can be moved vertically by pneumatic operation.

helmet is made of fiberglass reinforced plastic (FRP), and the sensor helmet with gradiometers mounted is shown in figure 2 . The distance between the neighboring gradiometers is about $35 \mathrm{~mm}$. The gradiometers were arranged to measure the field component vertical to the helmet surface.

All the signal wires were low thermal conductivity Manganin wires of $0.127 \mathrm{~mm}$ diameter. For the mechanical support of the insert, FRP tubes were used for mechanical rigidity and for the low thermal load. At the neck part of the insert, multiple layers of Styrofoam/Cu-PCB were used for the radiation baffle. The helmet dewar is a reservoir type with a reservoir volume of 881 . The inner tail diameter is $400 \mathrm{~mm}$. The average boil-off rate of liquid $\mathrm{He}$ is $101 \mathrm{~d}^{-1}$ when the MEG system is in operation every day, and the refill interval of liquid He is 1 week.

\subsection{Readout and control electronics}

The fabricated DROS gradiometers had maximum modulation voltages of about $110 \mu \mathrm{V}$, and the flux-to-voltage transfers are typically about $1 \mathrm{mV} / \Phi_{0}$ at the optimum modulation amplitudes of about $70 \mu \mathrm{V}$. To detect the DROS output voltages directly, preamplifier circuits were made by using common operational amplifiers (LT1128; Linear Technology). When the LT1128 operational amplifiers were configured in instrumentation amplifier mode, the input voltage noise of 


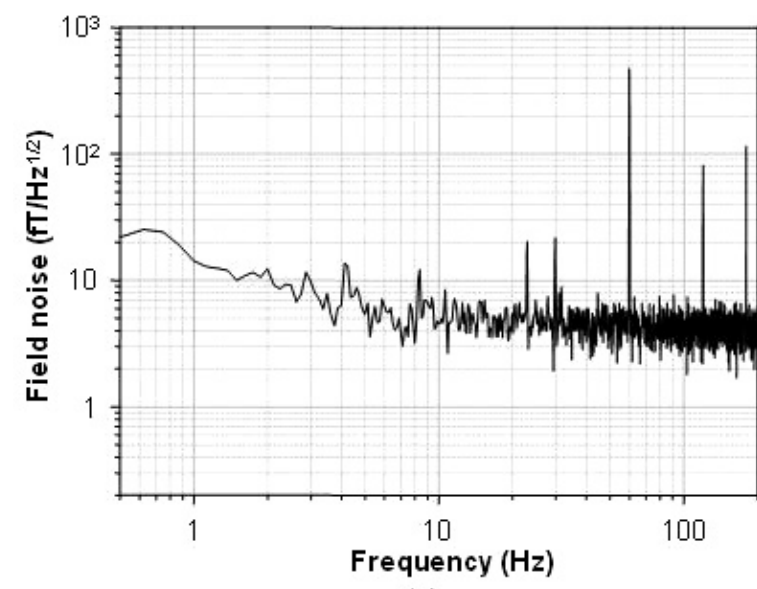

(a)

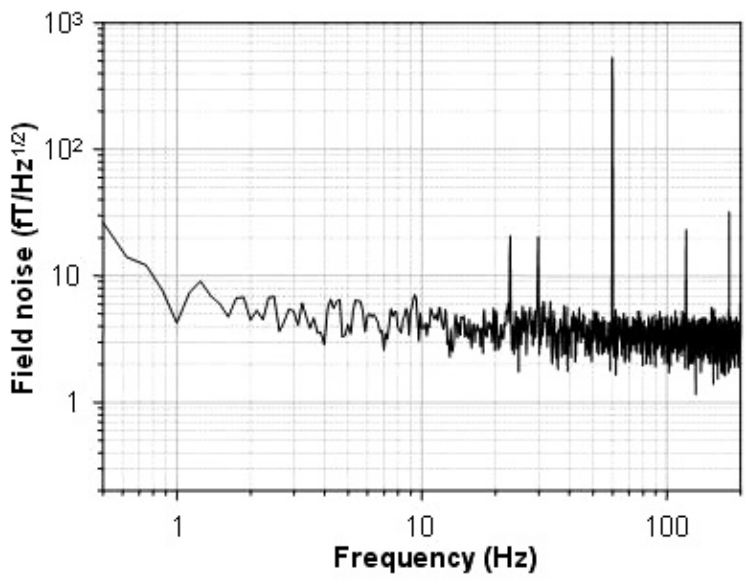

(b)

Figure 4. Magnetic field noise spectra of two arbitrary gradiometers. (a) Noise spectrum of channel number 3 (positioned at the right frontal of the helmet) and (b) channel number 116 (left occipital).

the preamplifier was $1.5 \mathrm{nV} \mathrm{Hz}^{-1 / 2}$ at $100 \mathrm{~Hz}$. With a typical flux-to-voltage transfer of $1 \mathrm{mV} / \Phi_{0}$, the contribution of preamplifier noise is $1.5 \mu \Phi_{0} \mathrm{~Hz}^{-1 / 2}$ at $100 \mathrm{~Hz}$, or equivalent field noise of $0.7 \mathrm{fT} \mathrm{Hz}^{-1 / 2}$ at $100 \mathrm{~Hz}$ for the field-to-flux transfer coefficient of the flux transformer $\left(0.46 \mathrm{nT} / \Phi_{0}\right)$, which seems a practically negligible contribution to the total noise of the MEG system.

FLL circuits operate in DC mode only (DC current bias and DC amplifier), and modulation of, for example, the magnetic flux was not used. The PCB board of each FLL circuit has a size of $45 \mathrm{~mm} \times 95 \mathrm{~mm}$, and 16 FLL PCBs were assembled in an aluminum box having a size of $182 \mathrm{~mm}(W) \times$ $48 \mathrm{~mm}(H) \times 123 \mathrm{~mm}(D)$. In total, eight aluminum boxes were mounted on top of the gantry for 128-channel operation. Analog signal processing (ASP) consists of a high-pass filter $(0.01 \mathrm{~Hz})$, low-pass filter $(100 \mathrm{~Hz})$, notch filter $(60 \mathrm{~Hz})$, and amplifier (100 times). Each ASP PCB board has four ASP channels. ASP circuits were installed outside the MSR. The control of FLL and ASP operations was done by digital control signals which set the SQUID bias current, voltage offset of the integrator, flux bias, FLL switch, and filter switches. The digital pulses were transmitted through a fiber-optic cable.

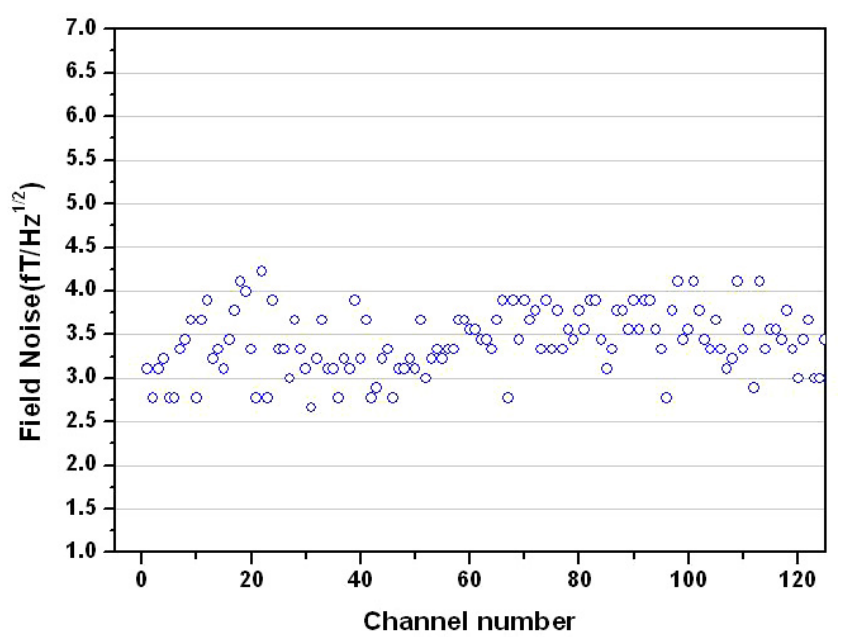

Figure 5. Magnetic field noise level as a function of channel number. The field noise value was measured at $100 \mathrm{~Hz}$.

\subsection{Magnetically shielded room}

The MSR is three-shell structure with two layers of Permalloy (each $2.1 \mathrm{~mm}$ thick) and one layer of aluminum $(12 \mathrm{~mm}$ thick). The total thickness of the wall, including the finishing wooden plates, is $25 \mathrm{~cm}$. Aluminum H-beam frames were used for the mechanical construction of the room. Thin (0.35 mm thick) Permalloy plates were overlapped using an adhesive tape to form $2.1 \mathrm{~mm}$ thick plates. Generally, the thinner Permalloy plate has higher permeability than the thick one [19]. Experience from constructions of several MSRs also showed that thinner Permalloy plates have higher permeability than thick ones (typically $1 \mathrm{~mm}$ ), and the total weight of Permalloy material can be reduced by using multiple layers of thinner Permalloy plates. As estimated from the shielding factors of several MSRs at low frequency $(0.01 \mathrm{~Hz})$, a Permalloy plate of $0.35 \mathrm{~mm}$ thickness had a permeability of about 40000 , while a thick one ( $1 \mathrm{~mm}$ thick) had a permeability of about 25000 . The inner dimensions of the fabricated MSR are $2.4 \mathrm{~m} \times 2.4 \mathrm{~m} \times 2.4 \mathrm{~m}$. The average shielding factors along the three directions are $45 \mathrm{~dB}$ at $0.1 \mathrm{~Hz}$ and $80 \mathrm{~dB}$ at $10 \mathrm{~Hz}$. The earth DC magnetic field magnitude was attenuated by about $60 \mathrm{~dB}$.

\section{Operation of the system}

The helmet dewar was mounted on a nonmagnetic gantry, as shown in figure 3 . The gantry allows vertical movement, and tilting of the dewar up to $30^{\circ}$ from vertical. The subject chair also allows vertical movement using pneumatic operation. The magnetic field noise spectra of the system were measured without a subject inside the MSR. Figure 4 shows two arbitrary noise spectra from the 128 channels. Some peaks between 10 and $30 \mathrm{~Hz}$ are caused by mechanical vibration of the building and insert. The distribution of field noises depending on the channel number is shown in figure 5. The system noise level is in the range 2.7-4.2 $\mathrm{fT}_{\mathrm{rms}} \mathrm{Hz}^{-1 / 2}$, with an average level of about $3.5 \mathrm{fT}_{\mathrm{rms}} \mathrm{Hz}^{-1 / 2}$ at $100 \mathrm{~Hz}$. 


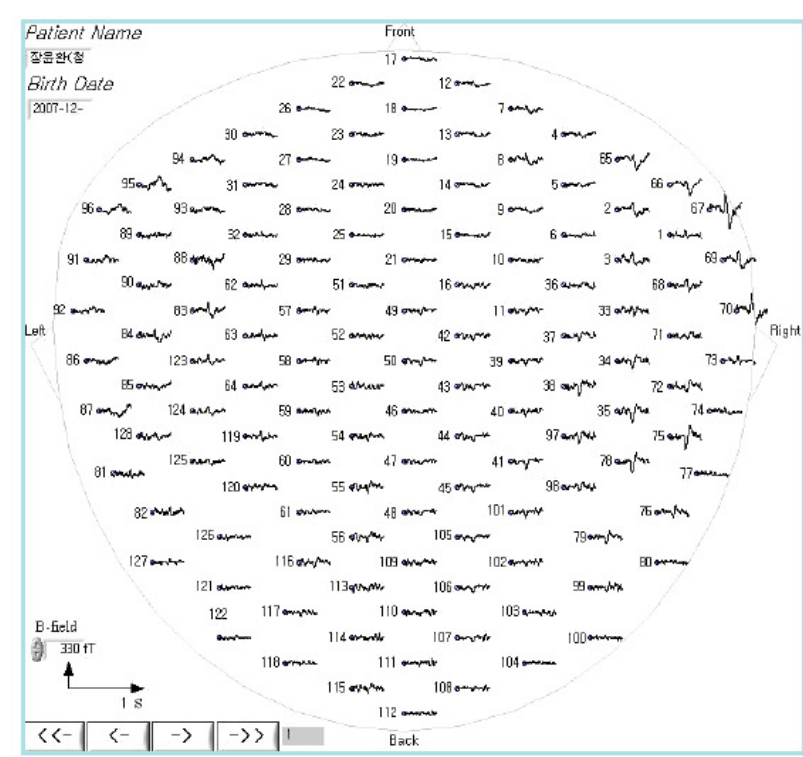

(a)

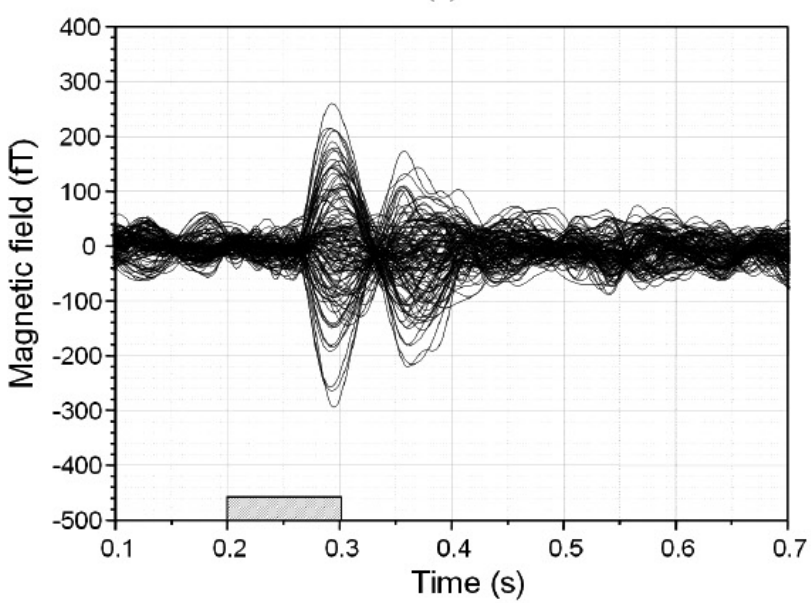

(b)

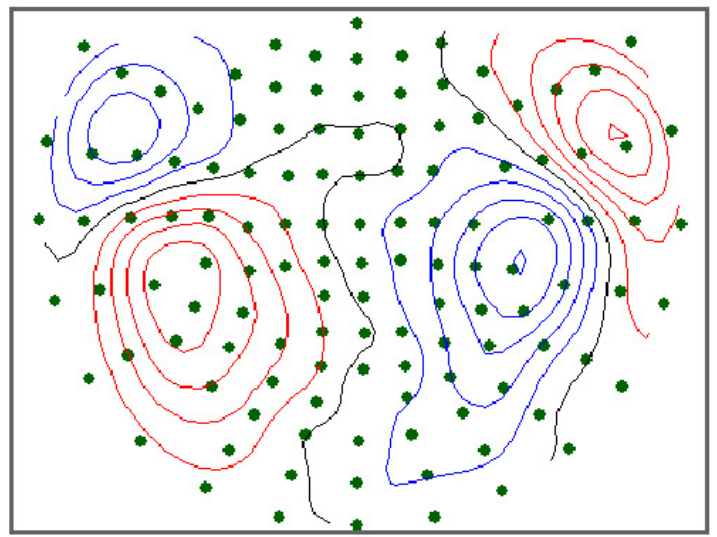

(c)

Figure 6. Auditory-evoked response. (a) Waveform distribution of the auditory-evoked field, (b) overlapping of the 128 channels, and (c) magnetic field map at $100 \mathrm{~ms}$ after stimulus onset. The hatched box on the time axis of (b) is the stimulus duration. 100 epochs were averaged, and $40 \mathrm{fT}$ interval in the contour map (c).

MEG signals were measured using two 80-channel, 16-bit A/D cards (National Instruments, PCI-6255) with a maximum sampling rate of $5 \mathrm{kHz}$. A Windows-based personal computer was used for the measurements and analyses of the MEG data. As a test of the system operation, auditory-evoked fields were measured. Auditory stimuli of a $1 \mathrm{kHz}$ tone burst, $100 \mathrm{~ms}$ duration, and about $60 \mathrm{~dB}$ normal hearing level were applied to the right ear of a normal subject in random intervals of 1-2 s, and the magnetic fields were measured at a sampling rate of $1 \mathrm{kHz}$. Figure 6(a) shows the measured auditory-evoked signal distribution. The signals were digitally processed by averaging of 100 epochs, and $40 \mathrm{~Hz}$ low-pass filtering. Figure 6(b) shows the overlapped waveforms. A clear N100 m peak, the magnetic field peak occurring at $100 \mathrm{~ms}$ from the onset of stimulus, can be seen. Mapping of the N100 m component is shown in figure 6(c). The field map pattern looks like a typical dipolar pattern, showing the successful measurement of the auditory response.

\section{Conclusions}

A whole-head MEG system was developed having features of compact structure of the axial gradiometer and compact FLL circuit. By applying a simple process for the superconductive connection between the pickup coil and the input coil, the mechanical structure of the superconductive connection became very compact. Furthermore, by positioning the SQUID sensor close to the compensation coil, the total length of the gradiometer became short, resulting in an increase of the liquid He refilling period. As a novel approach to increase the flux-tovoltage transfer of the SQUID, a DROS having flux-to-voltage transfer of about $1 \mathrm{mV} / \Phi_{0}$ was used, so that the contribution of preamplifier input noise became negligible in direct readout mode. After integrating the 128-channel gradiometer insert into the helmet dewar, the boil-off of liquid He was about $101 \mathrm{~d}^{-1}$, with a refill interval of 1 week. The fabricated system was operated inside a moderately MSR, and the average magnetic field noise level of the system was $3.5 \mathrm{fT}_{\mathrm{rms}} \mathrm{Hz}^{-1 / 2}$ in the white region. Auditory-evoked responses were measured, showing a successful demonstration of the developed MEG system.

\section{References}

[1] Hämäläinen M, Hari R, Ilmoniemi R J, Knuutila J and Lounasmaa O V 1993 Magnetoencephalography. Theory, instrumentation and applications to the noninvasive study of human brain function Rev. Mod. Phys. 65 413-97

[2] Wheless J W, Castillo E, Maggio V, Kim H L, Breier J I, Simos P G and Papanicolaou A C 2004 Magnetoencephalography (MEG) and magnetic source imaging (MSI) Neurologist 10 138-53

[3] Knuutila J 2007 Instrumentation development: from MEG recording to functional mapping Int. Congr. Ser. 1300 7-10

[4] Del Gratta C, Pizzella V, Tecchio F and Romani G L 2001 Magnetoencephalography - a noninvasive brain imaging method with $1 \mathrm{~ms}$ time resolution Rep. Prog. Phys. 64 1759-814

[5] Nowak H 1998 Magnetism in Medicine ed W Andrä and H Nowak (Berlin: Wiley) pp 85-135

[6] Vrba J and Robinson S E 2002 SQUID sensor array configurations for magnetoencephalography applications Supercond. Sci. Technol. 15 R51-89 
[7] Ketchen M B 1987 Integrated thin-film dc SQUID sensors IEEE Trans. Magn. 23 1650-7

[8] Kiryu S, Ogashiwa T, Chinone K, Kasai N, Kado H, Ishikawa N, Nakanishi M and Koyanagi M 1989 Pb-In wire bonding for superconducting connection between thin-film DC-SQUID and pickup coil Advances in Biomagnetism (New York: Plenum) pp 653-6

[9] ter Brake H J M, Flokstra J, Houwman E P, Veldhuis D, Jaszczuk W, Stammis R, van Ancum G K and Rogalla H 1992 On the SQUID-module for the UT multichannel neuromagnetometer Superconducting Devices and Their Applications (Berlin: Springer) pp 521-4

[10] Dössel O, David B, Fuchs M, Krüger J, Lüdeke K M and Wischmann H 1993 A modular 31-channel SQUID system for biomagnetic measurements IEEE Trans. Appl. Supercond. A 3 1883-6

[11] Drung D 1996 SQUID Sensors: Fundamentals, Fabrication and Applications ed $\mathrm{H}$ Weinstock (Dordrecht: Kluwer) pp 63-116

[12] Pizzella V, Della Penna S, Del Gratta C and Romani G L 2001 SQUID systems for biomagnetic imaging Supercond. Sci. Technol. 14 R79-114
[13] Adelerhof D J, Nijstad H, Flokstra J and Rogalla H 1994 (Double) relaxation oscillation SQUIDs with high flux-to-voltage transfer: simulations and experiments J. Appl. Phys. 76 3875-86

[14] Lee Y H, Yu K K, Kim J M, Kwon H, Kim K and Park Y K 2008 64-channel second-order axial gradiometer system based on DROS for magnetocardiogram in a thin shielded room Physica C 468 1942-5

[15] Lee Y H, Kim J M, Kim K, Kwon H, Yu K K, Kim I S and Park Y K 2006 64-channel magnetocardiogram system based on double relaxation oscillation SQUID planar gradiometers Supercond. Sci. Technol. 19 S284-8

[16] Lee Y H, Kwon H C, Kim J M, Park Y K and Park J C 1998 Double relaxation oscillation SQUID with reference junction for biomagnetic multi-channel applications Appl. Supercond. 5 413-8

[17] ter Brake H J M, Fleuren F H, Ulfman J A and Flokstra J 1986 Elimination of flux-transformer crosstalk in multichannel SQUID magnetometers Cryogenics 26 667-70

[18] Jaszczuk W, ter Brake H J M, Flokstra J, Veldhuis D, Stammis R and Rogalla H 1991 Bonding of a niobium wire to a niobium thin film Meas. Sci. Technol. 2 1121-2

[19] http://www.mumetal.com or http://www.mushield.com 